\title{
Experimental study of subwavelength focusing by left- handed metamaterials with a negative refractive index
}

\author{
Ekmel Ozbay" and Koray Aydin
}

Nanotechnology Research Center, Department of Physics, and Department of Electrical and

Electronics Engineering, Bilkent University, Bilkent, 06800 Ankara, Turkey

ozbay@bilkent.edu.tr

\begin{abstract}
Two dimensional (2D) left-handed metamaterials (LHM) exhibiting negative refraction and subwavelength imaging are studied experimentally. A left-handed transmission band is observed at the frequencies where both dielectric permittivity and magnetic permeability are simultaneously negative. 2D LHM structure is verified to have a negative refractive index by measuring the refraction through a prism-shaped sample. Subwavelength focusing is observed by using a flat LHM superlens.
\end{abstract}

Keywords: left-handed metamaterial, negative refractive index, superlens.

\section{INTRODUCTION}

In recent years, left-handed metamaterials (LHMs) have become a remarkable research area and have received a considerable amount of interest due to their exotic electromagnetic properties that are not attainable from common materials. The left-handed media phenomenon was brought to the attention of the scientific community by Veselago four decades ago [1], and received great attention in recent years since the first experimental demonstration of LHMs by Smith et al. [2]. Ubiquitous materials have positive refractive indices, however, it is possible to obtain negative values of refractive index by making use of the concept of metamaterials [3] and photonic crystals [4] .

Ordinary materials with a positive refractive index always require curved surfaces to focus EM waves. Positive-index lenses suffer from the diffraction limit that is dictated by wave optics and can only focus objects with sizes on the order of a half-wavelength. The finer details of the image are carried by high- $k$ components, the so called evanescent waves and quickly decay before reaching the image plane. Therefore, the contribution of evanescent components to the resolution of the image is absent in conventional lenses. However, a parallel-sided slab lens constructed from a negative index material brings EM waves into focus $[1,5]$. Double focusing occurs inside and outside the lens, which is easily deducible from a simple ray diagram. Such lenses are also called flat lenses because their surfaces are not curved. Negative index materials can restore the amplitude of evanescent waves and therefore enable subwavelength focusing [5].

In this paper, we present experimental evidence of negative refraction and subwavelength focusing in left-handed materials. The negative index of refraction for the two-dimensional (2D) LHM is verified by measuring the refraction from a prism-shaped LHM structure. A point source is placed at two different distances away from the LHM lens, and clear images of the source are observed. The measured full widths at half maximum (FWHM) of the focused beams are $0.36 \lambda$ and $0.4 \lambda$ for two different source distances. This is consistent with the result that negative index materials can focus EM waves onto an area smaller than a wavelength. 


\section{LEFT-HANDED METAMATERIAL}

LHM structures are generally composed of two different structures, SRRs and thin wire grids. Periodic thin wire media is responsible for the negative effective permittivity, whereas periodic SRR structure provides negative effective permeability. The LHM studied in this work consists of the two-dimensional periodic arrangement of split-ring resonator (SRR) and thin wire arrays. The parameters of the 2D LHM structure could be found elsewhere [6].

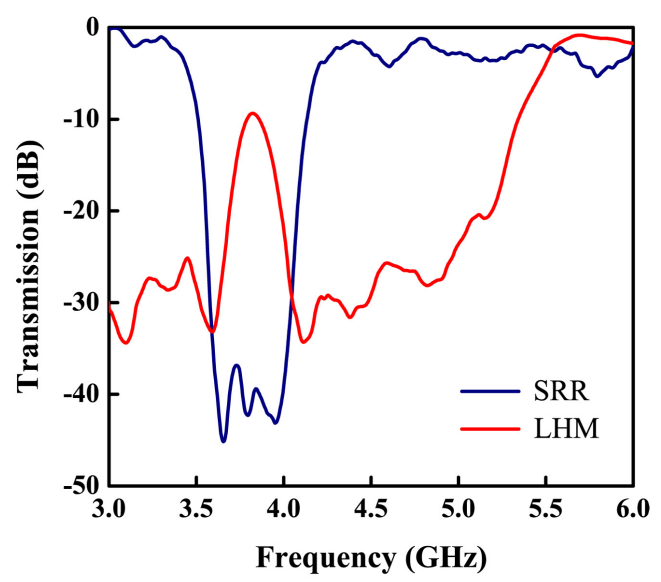

Fig. 1. Measured transmission spectra of periodic SRR (blue line) and LHM (red line) arrays.

We measured transmission and reflection spectra of 2D LHMs by using HP 8510C network analyzer system that is capable of measuring $\mathrm{S}$ parameters, transmission and reflection phase and group delay. In the measurements transmitter and receiver horn antennae are connected to the network analyzer. Figure 1 shows the measured transmission spectra of periodic SRR array (blue line) and periodic LHM structure (red line). The number of layers along the propagation direction is 5. A band gap is observed in the transmission spectrum of periodic SRR between 3.60 and $4.05 \mathrm{GHz}$. In an earlier work, we showed that this band gap is due to the magnetic resonance of SRRs and thus the permeability should have negative values at this specific frequency range [7]. The thin wire media has a plasma frequency at $8 \mathrm{GHz}$ [6]. Therefore, one should observe a transmission band within the frequency region where both permittivity and permeability are negative. As seen in Fig.1, a transmission band is observed between 3.65-4.05 GHz, where the effective permeability and effective permittivity of LHM are simultaneously negative. The peak value within this transmission band is $-9.9 \mathrm{~dB}$ at 3.86 GHz. Note that the second transmission band starts around $5.3 \mathrm{GHz}$ for the LHM structure. This is due to downward shift of the plasma frequency of the composite metamaterial. Adding SRR structure into the wire media affects the electric response of the system and causes a shift in the plasma frequency [7].

\section{NEGATIVE REFRACTION}

Although transmission measurements provide some information about the composite metamaterial of SRRs and wires, additional measurements must be done in order to assure that the metamaterial has left-handed characteristics. For this purpose, we performed experiments to verify the refractive index of the 2D LHM under investigation is indeed negative. Prism-shaped structures can be used to find the sign and the value of the refractive 
index [3,6]. We constructed a prism-shaped LHM with a prism angle of $\theta=26^{\circ}$. The first interface of left-handed material is excited with EM waves emanating from the transmitter horn antenna located at a distance of $13 \mathrm{~cm}(\sim 2 \lambda)$ away from the first interface of the prism. The receiver horn antenna is placed $70 \mathrm{~cm}(\sim 10 \lambda)$ away from the second interface of the sample and mounted on a rotating arm to scan the angular distribution of the refracted signal. Details of the experiments could be found in ref. 6 .

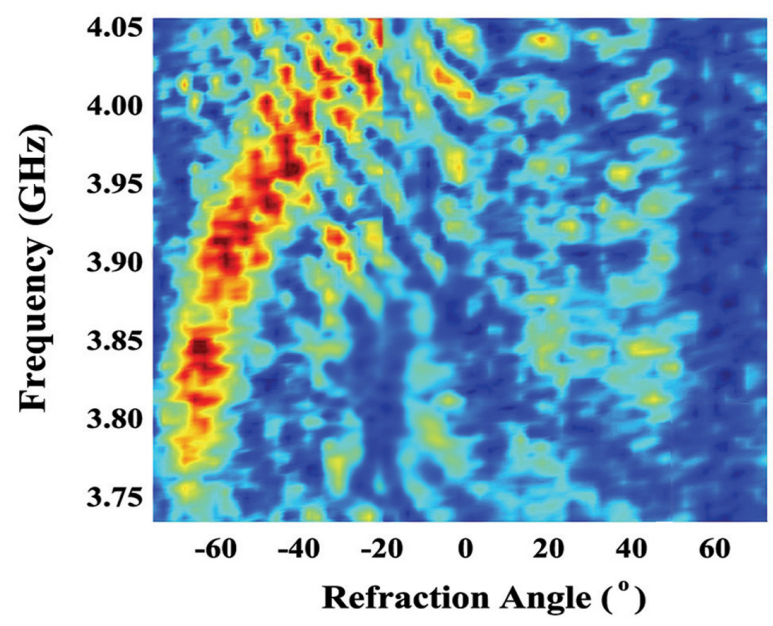

Fig. 2. Measured beam profiles of the EM waves refracted from a 2D prism shaped LHM as a function of frequency and angle of refraction between $3.73-4.05 \mathrm{GHz}$. The beam clearly refracted on the negative side of the normal, indicating a negative refraction behavior.

The measured field intensity at $70 \mathrm{~cm}$ away from the prism is plotted as a function of frequency and refraction angle in Fig. 2. It is evident from the figure that the transmitted beam is refracted on the negative side of the normal, so that the refraction angle is negative. Negative refraction angle could only be possible if the measured sample has a negative refractive index. This is easily deducible from the Snell's law which in our case could be written as $n_{\mathrm{LHM}} \sin \theta_{\mathrm{i}}=n_{\text {air }} \sin \theta_{\mathrm{r}}$. This measurement verifies that the refractive index values of the 2D LHM is negative between 3.73 and $4.05 \mathrm{GHz}$. At lower frequencies the EM waves are refracted at higher negative refraction angles, which results in a higher negative refractive index. The refraction index is lowered if we go to higher frequencies. The angle of the refraction at $3.86 \mathrm{GHz}$ is measured to be $\theta_{\mathrm{r}}=-60^{\circ}$. By using Snell's law, the refractive index is calculated as $n_{\mathrm{eff}}=-1.91 \pm 0.05$ at $3.86 \mathrm{GHz}$, where the maximum transmission is observed.

\section{SUBWAVELENGTH FOCUSING}

A slab-shaped lens, so called flat lens, may focus an omni-directional source [8]. Electromagnetic waves (EM) emerging from a point source placed near such a lens will first be refracted through the first air-LHM interface and will come into focus inside the material. Then outgoing EM waves will face refraction again at the second LHM-air interface and the refracted beam will meet the optical axis of flat lens, where the second focusing will occur. If the lens is not thick enough, the focusing may not occur inside the lens, which in turn will result in a diverging beam instead of a converging beam, even if the material is negatively refracting. Therefore, the thickness of the lens plays a crucial role for observing flat lens behavior. 
In the focusing measurements we used a LHM lens with 10 layers along the propagation direction. We used a monopole antenna to imitate the point source. A point source is a subwavelength feature and can not be focused smaller than a half wavelength with a positiveindex lens. The source is located away from the LHM lens at two different source distances of $d_{\mathrm{s}}=0.5 \lambda$ and $d_{\mathrm{s}}=\lambda$. The intensity distribution of an EM wave is scanned from the second LHM-air interface by another monopole antenna with $\Delta x=\Delta z=5 \mathrm{~mm}$ steps.

(a)

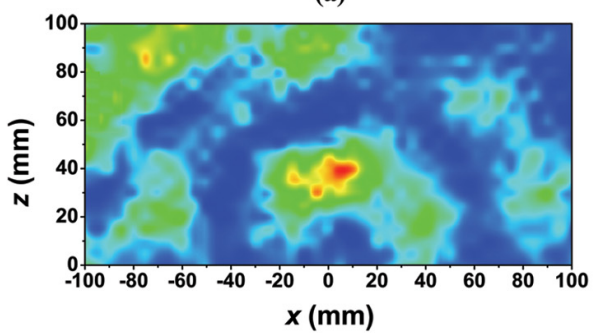

(b)

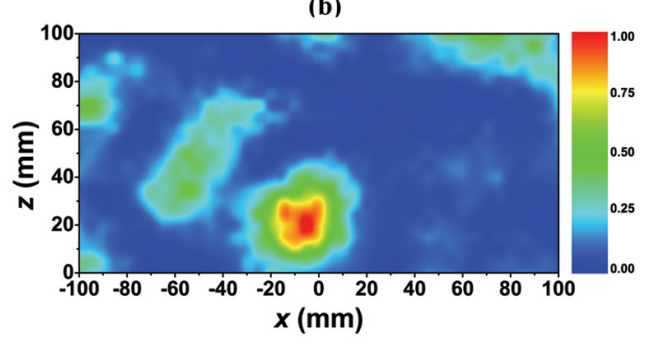

Fig. 3. Measured transmission spectra along the $x-z$ plane for a point source located at (a) $d_{\mathrm{s}}=0.5 \lambda$, and (b) $d_{\mathrm{s}}=\lambda$ away from the LHM lens. The $x$ direction is parallel to the LHM lens where $x=0$ is the optical axis of the flat lens, whereas the $z$ direction is perpendicular to the LHM lens where $z=0$ is the LHM-air interface.

The scanned field intensities are shown in Fig. 3. In the figure the intensities are normalized with respect to the maximum intensity values. The point $z=0$ corresponds to the LHM-air interface. Figure 3(a) provides the field intensity for the omni-directional source located at $d_{\mathrm{s}}=\lambda / 2$ away from the LHM lens. As seen in the figure, an image is formed at a focal length of $\mathrm{z}=40 \mathrm{~mm}$. The case where the point source is placed at $d_{\mathrm{s}}=\lambda$ is plotted in Fig. 3(b). The image is observed at $z=20 \mathrm{~mm}$ (Fig. 3(b)). As the point source is moved away from the LHM flat lens, the focal length is shifted towards the flat lens, which is consistent with imaging theory [8].

From the intensities along the lateral direction ( $x$ axis), FWHMs are found to be $0.4 \lambda$ and $0.36 \lambda$ (not plotted here) for the source distances $d_{\mathrm{s}}=\lambda / 2$ and $d_{\mathrm{s}}=\lambda$, respectively, which are below the diffraction limit. Since the material used to construct the flat lens has a negative refractive index, the evanescent waves are enhanced inside the lens subsequently enabling sub-wavelength imaging [5]. When the focal length is closer to the lens, the contribution of the evanescent waves to focusing is higher, which in turn results in better subwavelength resolution $(0.36 \lambda)$.

First experimental demonstrations of metamaterials are achieved at microwave frequencies. We discussed the imaging performance of negative-index metamaterials at microwave frequencies. The negative-index metamaterials are recently demonstrated at optical frequencies [9]. Subwavelength imaging is still achievable even in the absence of negative-index. In the near-field regime, the electrostatic and magnetostatic limits apply, and therefore, the electric and magnetic responses of materials can be treated as decoupled. This in turn brings the possibility of constructing superlenses from materials with negative permittivity or negative permeability. Although the negative-index metamaterials are still under development at optical frequencies, the negative permittivity is easily achievable at optical frequencies. Silver superlenses are shown to exhibit subwavelength imaging behavior at optical frequencies [10,11]. SiC layers could also be used for imaging sub-diffractionlimited objects [12]. The recovery of evanescent waves in these superlenses is due to the excitation of surface plasmons [10]. These optical superlenses operate at the near-field region since the image is formed near the superlens. If the image forms far away from the superlens, 
the enhanced evanescent waves would decay rapidly after passing the superlens-air interface and their contribution to the resolution would not be enough to resolve subwavelength features. However Z. Liu et al., recently developed a far-field superlens which makes it possible to obtain subwavelength resolution at the far-field region [13]. They used subwavelength gratings to enhance the evanescent waves and to convert the evanescent modes to the propagating modes. The images of subwavelength features are then collected far from the superlens [13]. Z. Liu et al., also demonstrated another type of lens, so called hyperlens to magnify the features smaller than a half-wavelength [14]. They used cylindrical shaped silver and alumina layers to construct the hyperlens which is an anisotropic medium with hyperbolic dispersion.

The advantage of using negative-index lenses over negative-permittivity or negativepermeability lenses is that the subwavelength resolution can be obtained for both transverseelectric (TE) and transverse-magnetic (TM) polarization of EM waves. However, singlenegative lenses can only focus EM waves with one particular polarization. Since the negativeindex metamaterials are gearing towards optical frequencies [9], we believe that subwavelength focusing can be achieved at visible wavelengths. However, meticulous designs are needed to achieve low-loss, impedance-matched superlenses at optical frequencies, since the amount of absorption losses is relatively large compared to the losses at microwave frequencies.

\section{CONCLUSION}

We studied transmission, refraction and imaging of a two-dimensional left-handed metamaterial experimentally. We observed a band gap at the transmission spectrum of SRR array which is due to the negative magnetic permeability. A left-handed transmission band is obtained when a wire array is added at the back side of SRR array. The transmission band appeared at a frequency regime where both the effective dielectric permittivity and magnetic permeability of the LHM are simultaneously negative. By measuring the refraction through a prism-shaped LHM, we verified that the structure has a negative refractive index. Subwavelength focusing measurements are performed by using monopole antennae as point sources. The flat lens behavior of a 2D LHM slab is studied for sources located at two different distances away from the air-LHM interface.

\section{Acknowledgment}

This work is supported by the European Union under the projects EU-NoEMETAMORPHOSE, EU-NoE-PHOREMOST, and TUBITAK under Projects Nos. 104E090, 105E066, 105A005, and 106A017. One of the authors (E.O.) also acknowledges partial support from the Turkish Academy of Sciences.

\section{References}

[1] V. G. Veselago, "The electrodynamics of substances with simultaneously negative values of permittivity and permeability," Sov. Phys. Usp. 10, 509 (1968).

[2] D. R. Smith, W. J. Padilla, D. C. Vier, S. C. Nemat-Nasser, and S. Schultz, "Composite medium with simultaneously negative permeability and permittivity," Phys. Rev. Lett. 84, 4184-4187 (2000) [doi:10.1103/PhysRevLett.84.4184].

[3] R. A. Shelby, D. R. Smith, and S. Schultz, "Experimental verification of a negative index of refraction," Science 292, 77-79 (2001) [doi:10.1126/science.1058847]. 
[4] E. Cubukcu, K. Aydin, E. Ozbay, S. Foteinopoulou, and C. M. Soukoulis, "Electromagnetic waves: Negative refraction by photonic crystals," Nature 423, 604605 (2003) [doi:10.1038/423604b].

[5] J. B. Pendry, "Negative refraction makes a perfect lens," Phys. Rev. Lett. 85, 39663969 (2000) [doi:10.1103/PhysRevLett.85.3966].

[6] K. Aydin, K. Guven, C. M. Soukoulis, and E. Ozbay, "Observation of negative refraction and negative phase velocity in left-handed metamaterials," Appl. Phys. Lett. 86, 124102 (2005) [doi:10.1063/1.1888051].

[7] K. Aydin, K. Guven, M. Kafesaki, L. Zhang, C. M. Soukoulis, and E. Ozbay, "Experimental observation of true left-handed transmission peak in metamaterials," Opt. Lett. 29, 2623-2625 (2004) [doi:10.1364/OL.29.002623].

[8] K. Aydin, I. Bulu, and E. Ozbay, "Focusing of electromagnetic waves by a left-handed metamaterial flat lens," Opt. Exp. 13, 8753-8759 (2005) [doi:10.1364/OPEX.13.008753].

[9] V. M. Shalaev, "Optical negative-index metamaterials," Nature Photon. 1, 41-48 (2007) [doi:10.1038/nphoton.2006.49].

[10] N. Fang, H. Lee, C. Sun, and X. Zhang, "Sub-diffraction-limited optical imaging with a silver superlens," Science 308, 534-537 (2005). [doi: 10.1126/science.1108759]

[11] D. O. S. Melville, R. J. Blaikie, "Super-resolution imaging through a planar silver layer," Opt. Exp. 13, 2127 (2005) [doi:10.1364/OPEX.13.002127].

[12] T. Taubner, D. Korobkin, Y. Urzhumov, G. Shvets, R. Hillenbrand, "Near-field microscopy through a SiC superlens," Science 313, 1595 (2006) [doi:10.1126/science.1131025].

[13]Z. Liu, S. Durant, H. Lee, Y. Pikus, N. Fang, Y. Xiong, C. Sun, and X. Zhang, "Farfield optical superlens," Nano Lett. 7, 403, (2007) [doi:10.1021/n1062635n].

[14]Z. Liu, H. Lee, Y. Xiong, C. Sun and X. Zhang, "Far-Field optical hyperlens magnifying sub-diffraction-limited objects," Science 315, 1686, (2007) [doi:10.1126/science.1137368]. 